\title{
Lower order terms for the one-level densities of symmetric power $L$-functions in the level aspect
}

by

Guillaume Ricotta (Bordeaux) and Emmanuel Royer (Aubière)

\section{Introduction and statement of the result}

1.1. Description of the families of $L$-functions studied. The purpose of this paper is to compute the lower order terms of some particular statistics associated to low-lying zeros of several families of symmetric power $L$-functions in the level aspect: the one-level densities. As shown in [22], the main terms for the one-level densities are basically independent of the arithmetic of the families: they only rely on their random matrix theory type. For example, the following families have symplectic random matrix theory type: symmetric $r$ th power $L$-functions with $r$ even, $L$-functions of ideal class group of imaginary quadratic fields $\mathbb{Q}(\sqrt{-D})$ (with squarefree $D$ and $D$ congruent to -1 modulo 4$), L$-functions of quadratic Dirichlet characters, Rankin-Selberg $L$-functions (see 21] for a convincing manifestation of the power of prediction of random matrix theory in analytic number theory). However, lower order terms allow distinguishing these families [22, 6, 19]. We give a short description of the families under consideration. To any primitive holomorphic cusp form $f$ of prime level $q$ and even weight $\left({ }^{1}\right) \kappa \geqslant 2$ (see [22, $\S 2.1]$ for the automorphic background) say $f \in H_{\kappa}^{*}(q)$, one can associate its $r$ th symmetric power $L$-function denoted by $L\left(\operatorname{Sym}^{r} f, s\right)$ for any integer $r \geqslant 1$. It is given by the following absolutely convergent and non-vanishing Euler product of degree $r+1$ on $\operatorname{Re} s>1$ :

$$
L\left(\operatorname{Sym}^{r} f, s\right):=\prod_{p \in \mathscr{P}} L_{p}\left(\operatorname{Sym}^{r} f, s\right)
$$

2010 Mathematics Subject Classification: 11F66, 11M41, 15A52.

$K e y$ words and phrases: symmetric power $L$-functions, zeros, random matrix theory, onelevel densities, lower order terms.

$\left({ }^{1}\right)$ In this paper, the weight $\kappa$ is a fixed even integer and the level $q$ goes to infinity through the prime numbers. 
where

$$
L_{p}\left(\operatorname{Sym}^{r} f, s\right):=\prod_{i=0}^{r}\left(1-\frac{\alpha_{f}(p)^{i} \beta_{f}(p)^{r-i}}{p^{s}}\right)^{-1}
$$

for any prime number $p$ (we write $p \in \mathscr{P}$ ). From now on, $\alpha_{f}(p), \beta_{f}(p)$ are the Satake parameters of $f$ at the prime number $p$ and $\left(\lambda_{f}(n)\right)_{n \geqslant 1}$ is its sequence of Hecke eigenvalues, which is arithmetically normalised: $\lambda_{f}(1)=1$ and $\left|\lambda_{f}(p)\right| \leqslant 2$ for any prime $p$. We also define [4, (3.16) and (3.17)] a local factor at $\infty$ which is given by a product of $r+1$ Gamma factors

$$
\Gamma_{\mathbb{R}}(s):=\pi^{-s / 2} \Gamma(s / 2),
$$

namely

$$
\begin{aligned}
& L_{\infty}\left(\operatorname{Sym}^{r} f, s\right) \\
& \quad:=\prod_{0 \leqslant a \leqslant(r-1) / 2} \Gamma_{\mathbb{R}}\left(s+\frac{(2 a+1)(\kappa-1)}{2}\right) \Gamma_{\mathbb{R}}\left(s+1+\frac{(2 a+1)(\kappa-1)}{2}\right)
\end{aligned}
$$

if $r$ is odd and

$$
L_{\infty}\left(\operatorname{Sym}^{r} f, s\right):=\Gamma_{\mathbb{R}}\left(s+\mu_{\kappa, r}\right) \prod_{1 \leqslant a \leqslant r / 2} \Gamma_{\mathbb{R}}(s+a(\kappa-1)) \Gamma_{\mathbb{R}}(s+1+a(\kappa-1))
$$

if $r$ is even, where

$$
\mu_{\kappa, r}:= \begin{cases}1 & \text { if } r(\kappa-1) / 2 \text { is odd }, \\ 0 & \text { otherwise. }\end{cases}
$$

The completed $L$-function is defined by

$$
\Lambda\left(\operatorname{Sym}^{r} f, s\right):=\left(q^{r}\right)^{s / 2} L_{\infty}\left(\operatorname{Sym}^{r} f, s\right) L\left(\operatorname{Sym}^{r} f, s\right)
$$

and $q^{r}$ is the arithmetic conductor. We will need some control on the analytic behaviour of this function. Unfortunately, such information is not currently known in all generality. We sum up our main assumption in the following statement.

Hypothesis Nice $(r, f)$. The function $\Lambda\left(\operatorname{Sym}^{r} f, s\right)$ is a completed $L$ function in the sense that it has the following nice analytic properties:

- it can be extended to a holomorphic function of order 1 on $\mathbb{C}$,

- it satisfies a functional equation of the shape

$$
\Lambda\left(\operatorname{Sym}^{r} f, s\right)=\varepsilon\left(\operatorname{Sym}^{r} f\right) \Lambda\left(\operatorname{Sym}^{r} f, 1-s\right)
$$

where the sign $\varepsilon\left(\operatorname{Sym}^{r} f\right)= \pm 1$ of the functional equation is given by

$$
\varepsilon\left(\operatorname{Sym}^{r} f\right):= \begin{cases}+1 & \text { if } r \text { is even, } \\ \varepsilon_{f}(q) \varepsilon(\kappa, r) & \text { otherwise }\end{cases}
$$

with 


$$
\varepsilon(\kappa, r):=i^{\left(\frac{r+1}{2}\right)^{2}(\kappa-1)+\frac{r+1}{2}}= \begin{cases}i^{\kappa} & \text { if } r \equiv 1(\bmod 8), \\ -1 & \text { if } r \equiv 3(\bmod 8), \\ -i^{\kappa} & \text { if } r \equiv 5(\bmod 8) \\ +1 & \text { if } r \equiv 7(\bmod 8)\end{cases}
$$

and $\varepsilon_{f}(q)=-\sqrt{q} \lambda_{f}(q)= \pm 1$.

Remark 1. Hypothesis $\operatorname{Nice}(r, f)$ is known for $r=1$ (E. Hecke [10, 11, 12]), $r=2$ thanks to the work of S. Gelbart and H. Jacquet [7] and $r=3,4$ from the works of H. Kim and F. Shahidi [16, 15, 14. In addition, Rankin/Selberg theory enabled H. Kim and F. Shahidi to prove the functional equation and the meromorphic continuation to $\mathbb{C}$ for $k=5, \ldots, 9$ and the holomorphy in $\operatorname{Re} s>1$ for $k=5, \ldots, 8$. Note that the holomorphy at $s=1$ is expected since a holomorphic cusp form of prime level and trivial character is not of CM type. Very recently, a proof of the non-vanishing and the holomorphy of $L\left(\operatorname{Sym}^{r} f, s\right)$ on $\operatorname{Re} s \geqslant 1$ for any primitive holomorphic cusp form $f$ of prime level $q$, trivial character and even weight $f$ and any $r \geqslant 1$ has been announced by Barnet-Lamb, Geraghty, Harris \& Taylor [1, Theorem B].

We aim at studying the lower order terms of the one-level density for the family of $L$-functions given by

$$
\bigcup_{q \in \mathscr{P}}\left\{L\left(\operatorname{Sym}^{r} f, s\right): f \in H_{\kappa}^{*}(q)\right\}
$$

for any integer $r \geqslant 1$ and for the corresponding signed subfamilies (see [22] for more details).

1.2. One-level densities of the families. The purpose of this work is to determine the lower order terms of the one-level densities associated to the above families of $L$-functions. Let us give the statement of our result, in which $\nu$ is a positive real number, $\Phi$ is an even Schwartz function whose Fourier transform $\widehat{\Phi}$ is compactly supported in $[-\nu,+\nu]$ (denoted by $\Phi \in$ $\left.\mathscr{S}_{\nu}(\mathbb{R})\right)$ and $f$ is a primitive holomorphic cusp form of prime level $q$ and even weight $\kappa \geqslant 2$ for which Hypothesis $\operatorname{Nice}(r, f)$ holds $\left({ }^{2}\right)$. We refer to $[22, \S 2.2]$ for the probabilistic background. Note that, thanks to the Fourier inversion formula, such a function $\Phi$ can be extended to an entire even function which satisfies

$$
\forall s \in \mathbb{C}, \quad \Phi(s) \ll_{n} \frac{\exp (\nu|\operatorname{Im} s|)}{(1+|s|)^{n}}
$$

$\left({ }^{2}\right)$ Note that we do not assume any Generalised Riemann Hypothesis for the symmetric power $L$-functions. 
for any integer $n \geqslant 0$ according to [25, Theorem 7.22]. The one-level density (relative to $\Phi$ ) of $\operatorname{Sym}^{r} f$ is defined by

$$
D_{1, q}[\Phi ; r](f):=\sum_{\rho, \Lambda\left(\operatorname{Sym}^{r} f, \rho\right)=0} \Phi\left(\frac{\log \left(q^{r}\right)}{2 i \pi}\left(\operatorname{Re} \rho-\frac{1}{2}+i \operatorname{Im} \rho\right)\right)
$$

where the sum is over all non-trivial zeros $\rho$ of $L\left(\operatorname{Sym}^{r} f, s\right)$ repeated with multiplicities. The asymptotic expectation of the one-level density is by definition

$$
\lim _{\substack{q \text { prime } \\ q \rightarrow+\infty}} \sum_{f \in H_{\kappa}^{*}(q)} \omega_{q}(f) D_{1, q}[\Phi ; r](f)
$$

where

$$
\omega_{q}(f):=\frac{\Gamma(\kappa-1)}{(4 \pi)^{\kappa-1}\langle f, f\rangle_{q}}
$$

is the harmonic weight $\left({ }^{3}\right)$ of $f$ and $\langle\cdot, \cdot\rangle_{q}$ is Petersson's inner product on the modular curve of level $q$. Before stating our result, we define some constants. Let $\theta$ stand for the first Chebyshev function

$$
\theta(t):=\sum_{\substack{p \in \mathscr{P} \\ p \leqslant t}} \log p
$$

The first constant is

$$
C_{\mathrm{PNT}}:=\left(1+\int_{1}^{+\infty} \frac{\theta(t)-t}{t^{2}} d t\right)
$$

The second constant is

$$
C:=\sum_{p \in \mathscr{P}} \frac{\log p}{p^{3 / 2}-p}
$$

The last one is

$$
C_{\infty}:=-(r+1) \log \pi+C_{\Gamma}
$$

where

$$
C_{\Gamma}:=\sum_{0 \leqslant a \leqslant(r-1) / 2}\left\{\frac{\Gamma^{\prime}}{\Gamma}\left(\frac{1}{4}+\frac{(2 a+1)(\kappa-1)}{4}\right)+\frac{\Gamma^{\prime}}{\Gamma}\left(\frac{1}{4}+\frac{1}{2}+\frac{(2 a+1)(\kappa-1)}{4}\right)\right\}
$$

if $r$ is odd and

$$
C_{\Gamma}:=\frac{\Gamma^{\prime}}{\Gamma}\left(\frac{1}{4}+\frac{\mu_{\kappa, r}}{2}\right)+\sum_{1 \leqslant a \leqslant r / 2}\left\{\frac{\Gamma^{\prime}}{\Gamma}\left(\frac{1}{4}+\frac{a(\kappa-1)}{2}\right)+\frac{\Gamma^{\prime}}{\Gamma}\left(\frac{1}{4}+\frac{1}{2}+\frac{a(\kappa-1)}{2}\right)\right\}
$$

$\left({ }^{3}\right)$ An explanation why this factor facilitates the computations, a way to remove it, and its impact on lower order terms can be found in 17 . 
if $r$ is even. Finally, let us define

$$
\delta_{2 \mid r}:= \begin{cases}1 & \text { if } r \text { is even, } \\ 0 & \text { otherwise. }\end{cases}
$$

Theorem A. Let $r \geqslant 1$ be any integer and $\varepsilon= \pm 1$. Assume that Hypothesis $\operatorname{Nice}(r, f)$ holds for any prime number $q$ and any primitive holomorphic cusp form of level $q$ and even weight $\kappa \geqslant 2$. Let

$$
\nu_{1, \max }\left(r, \kappa, \theta_{0}\right):=\left(1-\frac{1}{2\left(\kappa-2 \theta_{0}\right)}\right) \frac{2}{r^{2}}
$$

with $\theta_{0}=7 / 64$. If $\nu<\nu_{1, \max }\left(r, \kappa, \theta_{0}\right)$ then the asymptotic expectation of the one-level density is

$$
\begin{aligned}
& \sum_{f \in H_{\kappa}^{*}(q)} \omega_{q}(f) D_{1, q}[; r](f)=\left[\widehat{\Phi}(0)+\frac{(-1)^{r+1}}{2} \Phi(0)\right] \\
&+\left[C_{\infty}-2(-1)^{r} C_{\mathrm{PNT}}-2 \delta_{2 \mid r} C\right] \frac{\widehat{\Phi}(0)}{\log \left(q^{r}\right)}+O\left(\frac{1}{\log ^{3}\left(q^{r}\right)}\right) .
\end{aligned}
$$

REMARK 2. The main terms of the asymptotic expectation of these onelevel densities have already been found in [22, 9]. The new information is the lower order terms, namely terms of size $1 / \log \left(q^{r}\right)$. Note that the arithmetic of the family does not enter the main terms but surfaces in the lower order ones. These results give further evidence for the fact that while the main terms for the one-level densities for low-lying zeros of families of $L$-functions are essentially of random matrix theory type, the lower order terms really depend on the arithmetic of the family. Universality is broken through these lower order terms.

REMARK 3. As will be seen in the proof of Proposition 3.1, the constant $C_{\text {PNT }}$ does not come from specific properties of our family. It has already appeared in other families [26, 20]. According to [23],

$$
C_{\mathrm{PNT}}=-\gamma-\sum_{p \in \mathscr{P}} \frac{\log p}{p^{2}-p}=-\lim _{x \rightarrow+\infty}\left[\log x-\sum_{\substack{p \in \mathscr{P} \\ p \leqslant x}} \frac{\log p}{p}\right]
$$

where $\gamma$ is Euler-Mascheroni's constant. Note that the first series occurring in the above equation is very slowly convergent, hence it is not so useful to compute the value of this constant. It is shown in [5] that

$$
C_{\mathrm{PNT}}=-\gamma-\sum_{\substack{p \in \mathscr{P} \\ p \leqslant P}} \frac{\log p}{p^{2}-p}+\sum_{k>1} \mu(k)\left(\frac{\zeta^{\prime}(k)}{\zeta(k)}+\sum_{\substack{p \in \mathscr{P} \\ p \leqslant P}} \frac{\log p}{p^{k}-1}\right),
$$

where the Möbius summand is bounded by $P^{1-k}$. It is possible to evaluate $C_{\text {PNT }}$ with Pari-Gp [2]. For instance, using a method developed by Henri 
Cohen, David Broadhurst used 67 minutes on a $2.2 \mathrm{GHz}$ Opteron to evaluate 5000 decimal places with $P=6779$ [3]. We extract the following value:

$C_{\mathrm{PNT}}=-1.3325822757332208817658287760710277488384594890424$.

Remark 4. To compute $C$ we define $u_{0}(l)=1, u_{1}(l)=\#\{d \mid l: 3 \leqslant d \leqslant$ $l / 2\}$ and

$$
u_{k}(l)=\sum_{\substack{d \mid l \\ 3 \cdot 2^{k-1} \leqslant d \leqslant l / 2}} u_{k-1}(d)
$$

We obtain

$$
C=\sum_{l \geqslant 3}\left(-\frac{\zeta^{\prime}}{\zeta}\left(\frac{l}{2}\right) \sum_{k=0}^{K}(-1)^{k} u_{k}(l)\right)+(-1)^{K} \sum_{l \geqslant 3 \cdot 2^{K}} u_{K+1}(l) \sum_{p} \frac{\log p}{p^{l / 2}} .
$$

With Pari-Gp [2], we get (in a few seconds)

$$
C=2.4768363850223143869989006981742171092171056763516 .
$$

REMARK 5. Using the relations

$$
\frac{\Gamma^{\prime}}{\Gamma}(z)+\frac{\Gamma^{\prime}}{\Gamma}\left(z+\frac{1}{2}\right)=2 \frac{\Gamma^{\prime}}{\Gamma}(2 z)-2 \log 2,
$$

and

$$
\frac{\Gamma^{\prime}}{\Gamma}(z+n)=\frac{\Gamma^{\prime}}{\Gamma}(z)+\sum_{j=0}^{n-1} \frac{1}{z+j}
$$

together with the special values

$$
\frac{\Gamma^{\prime}}{\Gamma}(1)=-\gamma, \quad \frac{\Gamma^{\prime}}{\Gamma}\left(\frac{1}{4}\right)=-\gamma-\frac{\pi}{2}-3 \log 2, \quad \frac{\Gamma^{\prime}}{\Gamma}\left(\frac{3}{4}\right)=-\gamma+\frac{\pi}{2}-3 \log 2
$$

[8, (8.365.6), (8.365.3), (8.366.1), (8.366.3), (8.366.4)] we obtain

$C_{\infty}=2 \sum_{j=1}^{[r(\kappa-1)-1] / 2} \frac{1}{j}\left(\frac{r+1}{2}-\left\lceil\frac{2 j+1}{2(\kappa-1)}-\frac{1}{2}\right\rceil\right)-(r+1)(\gamma+\log 2+\log \pi)$

if $r$ is odd and

$C_{\infty}=4 \sum_{j=1}^{r(\kappa-1) / 2} \frac{1}{2 j-1}\left(\frac{r}{2}-\left\lceil\frac{j}{\kappa-1}\right\rceil+1\right)-(r+1)(\gamma+3 \log 2+\log \pi)-(-1)^{r / 2} \frac{\pi}{2}$

if $r$ is even, where $\lceil\cdot\rceil$ stands for the ceiling function. It follows that if $r$ is large enough ( $\kappa$ being fixed) or if $\kappa$ is large enough ( $r$ being fixed) the coefficient of the lower order term (i.e. the one of $\left.\widehat{\Phi}(0) / \log \left(q^{r}\right)\right)$ is positive. In particular, in this cases, the families under consideration have more zeros than what is predicted by the random matrix theory model. For small values 
Table 1. Some values of the lower order coefficient

\begin{tabular}{|c|c|c|c|c|c|c|c|c|}
\hline$k r$ & 1 & 3 & 5 & 7 & 11 & 13 & 33 & 35 \\
\hline 2 & -7.49 & -10.32 & -12.15 & -13.31 & -14.24 & -14.17 & -1.83 & 0.21 \\
\hline 4 & -5.49 & -6.15 & -5.80 & -4.77 & -1.31 & 0.95 & 35.26 & 39.50 \\
\hline$k$ & 2 & 4 & 6 & 8 & 12 & 14 & 32 & 34 \\
\hline 2 & -8.12 & -13.53 & -11.86 & -15.90 & -16.44 & -13.08 & -5.00 & 0.12 \\
\hline 4 & -5.98 & -9.22 & -5.35 & -7.20 & -3.35 & 2.19 & 30.04 & 37.37 \\
\hline
\end{tabular}

(see Table 1), the lower order term coefficient is negative, showing a lack of zeros compared to what is predicted by the random matrix theory model.

REMARK 6 . Note that $\theta_{0}=7 / 64$ is the best known approximation towards Ramanujan-Petersson-Selberg's conjecture (see [22, hypothesis $\mathrm{H}_{2}(\theta)$, p. 16]) thanks to the works of H. Kim, F. Shahidi and P. Sarnak ([15, 14]). The value $\theta=0$ is expected.

REMARK 7. It is clear from the proof of Theorem A that the same result holds for the signed families with the same restriction on the support as in [22].

REMARK 8 . The particular case $r=1$ has already been investigated in [17].

Notation. Recall that $\mathscr{P}$ stands for the set of prime numbers. The main parameter in this paper is a prime number $q$, called the level, which goes to infinity through $\mathscr{P}$. If $f$ and $g$ are $\mathbb{C}$-valued functions of $q \in \mathscr{P}$ then the notations $f(q) \ll_{A} g(q)$ or $f(q)=O_{A}(g(q))$ mean that $|f(q)|$ is smaller than a "constant" (which only depends on $A$ ) times $g(q)$ at least for $q$ a large enough prime number.

2. Chebyshev polynomials and Hecke eigenvalues. Recall that the general facts about holomorphic cusp forms can be found in [22, §2.1]. Let $p \neq q$ be a prime number and $f \in H_{\kappa}^{*}(q)$. Denote by $\chi_{\mathrm{St}}$ the character of the standard representation St of SU(2). By the work of Deligne, there exists $\theta_{f, p} \in[0, \pi]$ such that

$$
\lambda_{f}(p)=\chi_{\mathrm{St}}\left(\begin{array}{cc}
e^{i \theta_{f, p}} & 0 \\
0 & e^{-i \theta_{f, p}}
\end{array}\right) .
$$

Moreover, the multiplicativity relation reads

$$
\begin{aligned}
\lambda_{f}\left(p^{\nu}\right) & =\chi_{\operatorname{Sym}^{\nu}}\left(\begin{array}{cc}
e^{i \theta_{f, p}} & 0 \\
0 & e^{-i \theta_{f, p}}
\end{array}\right)=X_{\nu}\left(\chi_{\mathrm{St}}\left(\begin{array}{cc}
e^{i \theta_{f, p}} & 0 \\
0 & e^{-i \theta_{f, p}}
\end{array}\right)\right) \\
& =X_{\nu}\left(\lambda_{f}(p)\right)
\end{aligned}
$$


where $\chi_{\mathrm{Sym}^{\nu}}$ is the character of the irreducible representation $\mathrm{Sym}^{\nu} \mathrm{St}$ of $\mathrm{SU}(2)$ and the polynomials $X_{\nu}$ are defined by their generating series

$$
\sum_{\nu \geqslant 0} X_{\nu}(x) t^{\nu}=\frac{1}{1-x t+t^{2}}
$$

They are equivalently defined by

$$
X_{\nu}(2 \cos \theta)=\frac{\sin ((\nu+1) \theta)}{\sin \theta} .
$$

These polynomials are known as Chebyshev polynomials of the second kind. Each $X_{\nu}$ has degree $\nu$, is even if $\nu$ is even and odd otherwise. The family $\left(X_{\nu}\right)_{\nu \geqslant 0}$ is a basis for the polynomial vector space $\mathbb{Q}[T]$, orthonormal with respect to the inner product

$$
\langle P, Q\rangle_{\mathrm{ST}}:=\frac{1}{\pi} \int_{-2}^{2} P(x) Q(x) \sqrt{1-\frac{x^{2}}{4}} d x .
$$

The following proposition lists the properties of Chebyshev polynomials needed for this work.

Proposition 2.1.

- If $\varpi \geqslant 0$ is any integer then

$$
X_{r}^{\varpi}=\sum_{j=0}^{r \varpi} x(\varpi, r, j) X_{j}
$$

with

$$
x(\varpi, r, j):=\left\langle X_{r}^{\varpi}, X_{j}\right\rangle_{\mathrm{ST}}=\frac{2}{\pi} \int_{0}^{\pi} \frac{\sin ^{\varpi}((r+1) \theta) \sin ((j+1) \theta)}{\sin ^{\varpi-1} \theta} d \theta .
$$

In particular,

$$
x(\varpi, r, j)= \begin{cases}0 & \text { if } j \equiv r \varpi+1(\bmod 2), \\
\frac{\left(\begin{array}{c}
\varpi \\
\varpi / 2
\end{array}\right)}{1+\varpi / 2} & \text { if } \varpi \text { is even, } r=1 \text { and } j=0 .\end{cases}
$$

- If $\alpha$ is a complex number of norm 1 and $n \geqslant 0$ is an integer then

$$
\alpha^{n}+\alpha^{-n}= \begin{cases}2 X_{0}\left(\alpha+\alpha^{-1}\right) & \text { if } n=0, \\ X_{1}\left(\alpha+\alpha^{-1}\right) & \text { if } n=1, \\ X_{n}\left(\alpha+\alpha^{-1}\right)-X_{n-2}\left(\alpha+\alpha^{-1}\right) & \text { otherwise. }\end{cases}
$$


- If $\alpha$ is a complex number of norm 1 and $r, n \geqslant 1$ are integers then

$$
\begin{aligned}
S(\alpha ; n, r) & :=\sum_{j=0}^{r} \alpha^{n(2 j-r)}=\delta_{2 \mid r}+\sum_{\substack{1 \leqslant j \leqslant r \\
j \equiv r(\bmod 2)}}\left[\alpha^{j n}+\alpha^{-j n}\right] \\
& =\sum_{\substack{0 \leqslant j \leqslant r \\
j \equiv r(\bmod 2)}}\left[X_{j n}\left(\alpha+\alpha^{-1}\right)-X_{j n-2}\left(\alpha+\alpha^{-1}\right)\right] \\
& =X_{r}\left(\alpha^{n}+\alpha^{-n}\right)
\end{aligned}
$$

where $X_{-1}=X_{-2}=0$ by convention.

- If $r \geqslant 1$ and $n \geqslant 1$ are integers then

$$
\sum_{\substack{0 \leqslant j \leqslant r \\ j \equiv r(\bmod 2)}}\left[X_{j n}-X_{j n-2}\right]=\sum_{j=0}^{r}(-1)^{j} X_{n-2}^{j} X_{n(r-j)}
$$

where $X_{-1}=X_{-2}=0$ by convention.

- If $l \geqslant 0$ is an integer then the expansion of $X_{l}$ in the canonical basis of $\mathbb{Q}[T]$ is

$$
X_{l}=\sum_{\substack{0 \leqslant u \leqslant l \\
u \equiv l(\bmod 2)}}(-1)^{(l-u) / 2}\left(\begin{array}{c}
(l+u) / 2 \\
u
\end{array}\right) T^{u} .
$$

Proof. The first point follows from the fact that $X_{r}^{\varpi}$ is a polynomial of degree $r \varpi$, which is even if $r \varpi$ is even and odd otherwise. Thus, (2.4) is the expansion of this polynomial in the orthonormal basis $\left(X_{j}\right)_{0 \leqslant j \leqslant r \varpi}$. The second point follows from the equality

$$
2 \cos (n \theta) \sin \theta=\sin ((n+1) \theta)-\sin ((n-1) \theta) .
$$

If $\alpha=\exp (i \theta)$ then this equality combined with $(2.3)$ leads to

$$
2 \cos (n \theta)=X_{n}(2 \cos \theta)-X_{n-2}(2 \cos \theta),
$$

which is the desired result since $2 \cos \theta=\alpha+\alpha^{-1}$ and $2 \cos (n \theta)=\alpha^{n}+\alpha^{-n}$. The third point is a direct consequence of the second one, of the direct computation

$$
S(\alpha ; n, r)=\frac{\alpha^{n(r+1)}-\alpha^{-n(r+1)}}{\alpha^{n}-\alpha^{-n}}
$$

and of

$$
X_{r}\left(\alpha^{n}+\alpha^{-n}\right)=X_{r}(2 \cos (n \theta))=\frac{\alpha^{n(r+1)}-\alpha^{-n(r+1)}}{\alpha^{n}-\alpha^{-n}}
$$

if $\alpha=\exp (i \theta)$. The fourth point is easily deduced from the fact that

$$
S(\alpha ; n, r)=\sum_{j=0}^{r}(-1)^{j} X_{n-2}^{j}\left(\alpha+\alpha^{-1}\right) X_{n(r-j)}\left(\alpha+\alpha^{-1}\right)
$$


for any complex number $\alpha$ of norm 1 . Let us prove the previous equality. According to [24, p. 727, first and second equations],

$$
\sum_{r \geqslant 0} X_{n r}\left(\alpha+\alpha^{-1}\right) t^{r}=\left[1+X_{n-2}\left(\alpha+\alpha^{-1}\right) t\right] \sum_{r \geqslant 0} X_{r}\left(\alpha^{n}+\alpha^{-n}\right) t^{r} .
$$

As a consequence,

$$
X_{n r}\left(\alpha+\alpha^{-1}\right)=X_{r}\left(\alpha^{n}+\alpha^{-n}\right)+X_{n-2}\left(\alpha+\alpha^{-1}\right) X_{r-1}\left(\alpha^{n}+\alpha^{-n}\right),
$$

which implies

$$
X_{r}\left(\alpha^{n}+\alpha^{-n}\right)=\sum_{j=0}^{r}(-1)^{j} X_{n-2}^{j}\left(\alpha+\alpha^{-1}\right) X_{n(r-j)}\left(\alpha+\alpha^{-1}\right) .
$$

The last point is obtained by developing 2.2 as an entire series in $x$.

3. Riemann's explicit formula for symmetric power $L$-functions. To study $D_{1, q}[\Phi ; r](f)$ for any $\Phi \in \mathscr{S}_{\nu}(\mathbb{R})$, we transform this sum over zeros into a sum over primes in the next proposition. In other words, we establish an explicit formula for symmetric power $L$-functions.

Proposition 3.1. Let $r \geqslant 1$ and $f \in H_{\kappa}^{*}(q)$ for which Hypothesis $\operatorname{Nice}(r, f)$ holds and let $\Phi \in \mathscr{S}_{\nu}(\mathbb{R})$. Then

$$
\begin{aligned}
D_{1, q}[\Phi ; r](f)= & {\left[\widehat{\Phi}(0)+\frac{(-1)^{r+1}}{2} \Phi(0)\right] } \\
& +\frac{\widehat{\Phi}(0)}{\log \left(q^{r}\right)}\left[C_{\infty}+2(-1)^{r+1} C_{\mathrm{PNT}}-2 \delta_{2 \mid r} C\right]+P_{q}^{1}[\Phi ; r](f) \\
& +\sum_{m=0}^{r-1}(-1)^{m} P_{q}^{2}[\Phi ; r, m](f)+P_{q}^{3}[\Phi ; r](f)+O\left(\frac{1}{\log ^{3}\left(q^{r}\right)}\right)
\end{aligned}
$$

where $C_{\mathrm{PNT}}$ is defined in (1.1), $C$ in 1.2), $C_{\infty}$ in (1.3) and

$$
\begin{aligned}
P_{q}^{1}[\Phi ; r](f) & :=-\frac{2}{\log \left(q^{r}\right)} \sum_{\substack{p \in \mathscr{P} \\
p \nmid q}} \lambda_{f}\left(p^{r}\right) \frac{\log p}{\sqrt{p}} \widehat{\Phi}\left(\frac{\log p}{\log \left(q^{r}\right)}\right), \\
P_{q}^{2}[\Phi ; r, m](f) & :=-\frac{2}{\log \left(q^{r}\right)} \sum_{\substack{p \in \mathscr{P} \\
p \nmid q}} \lambda_{f}\left(p^{2(r-m)}\right) \frac{\log p}{p} \widehat{\Phi}\left(\frac{2 \log p}{\log \left(q^{r}\right)}\right), \\
P_{q}^{3}[\Phi ; r](f) & :=-\frac{2}{\log \left(q^{r}\right)} \sum_{\substack{p \in \mathscr{P} \\
p \nmid q}} \sum_{n \geqslant 3}\left[\sum_{\substack{1 \leqslant j \leqslant r \\
j \equiv r(\bmod 2)}}\left(\lambda_{f}\left(p^{j n}\right)-\lambda_{f}\left(p^{j n-2}\right)\right)\right] \\
& \times \frac{\log p}{p^{n / 2}} \widehat{\Phi}\left(\frac{n \log p}{\log \left(q^{r}\right)}\right)
\end{aligned}
$$

for any integer $m \in\{0, \ldots, r-1\}$. 
Proof. Let

$$
G(s):=\Phi\left(\frac{\log \left(q^{r}\right)}{2 i \pi}\left(s-\frac{1}{2}\right)\right) .
$$

From [13, (4.11) and (4.14)] we get

$$
D_{1, q}[\Phi ; r](f)=\widehat{\Phi}(0)+C_{\infty} \frac{\widehat{\Phi}(0)}{\log \left(q^{r}\right)}+Q+O\left(\frac{1}{\log ^{3}\left(q^{r}\right)}\right)
$$

where

$$
Q:=-\frac{2}{\log \left(q^{r}\right)} \sum_{p \in \mathscr{P}} \sum_{n=1}^{+\infty}\left[\sum_{j=0}^{r} \alpha_{f}(p)^{j n} \beta_{f}(p)^{(r-j) n}\right] \widehat{\Phi}\left(\frac{n \log p}{\log \left(q^{r}\right)}\right) \frac{\log p}{p^{n / 2}} .
$$

Note that the contribution of the prime $q$ to $Q$ is given by

$$
-\frac{2}{r} \sum_{n=1}^{+\infty}\left(\frac{\lambda_{f}(q)^{r}}{\sqrt{q}}\right)^{n} \widehat{\Phi}\left(\frac{n}{r}\right) \ll \frac{1}{q^{(r+1) / 2}} .
$$

For $p \neq q$ we use

$$
\sum_{j=0}^{r} \alpha_{f}(p)^{j n} \beta_{f}(p)^{(r-j) n}=S\left(\alpha_{f}(p) ; n, r\right)
$$

with the notation of (2.7). We obtain

$$
S\left(\alpha_{f}(p) ; 1, r\right)=X_{r}\left(\alpha_{f}(p)+\alpha_{f}(p)^{-1}\right)=\lambda_{f}\left(p^{r}\right)
$$

according to 2.1 . This term contributes to $P_{q}^{1}[\Phi ; r](f)$. Then

$$
\begin{aligned}
S\left(\alpha_{f}(p) ; 2, r\right) & =\sum_{\substack{0 \leqslant j \leqslant r \\
j \equiv r(\bmod 2)}}\left[X_{2 j}\left(\alpha_{f}(p)+\alpha_{f}(p)^{-1}\right)-X_{2 j-2}\left(\alpha_{f}(p)+\alpha_{f}(p)^{-1}\right)\right] \\
& \left.=\sum_{j=0}^{r}(-1)^{j} X_{2(r-j)}\left(\alpha_{f}(p)+\alpha_{f}(p)^{-1}\right) \quad(\text { cf. } 2.8)\right) \\
& =\sum_{m=0}^{r-1}(-1)^{m} \lambda_{f}\left(p^{2(r-m)}\right)+(-1)^{r} .
\end{aligned}
$$

As a consequence,

$$
\begin{aligned}
Q= & P_{q}^{1}[\Phi ; r](f)+\sum_{m=0}^{r-1}(-1)^{m} P_{q}^{2}[\Phi ; r, m](f)-\frac{2}{\log \left(q^{r}\right)} S_{3} \\
& -\frac{2}{\log \left(q^{r}\right)} \sum_{\substack{p \in \mathscr{P} \\
p \nmid q}} \sum_{n \geqslant 3} S\left(\alpha_{f}(p) ; n, r\right) \frac{\log p}{p^{n / 2}} \widehat{\Phi}\left(\frac{\log \left(p^{n}\right)}{\log \left(q^{r}\right)}\right)+O\left(\frac{1}{q}\right)
\end{aligned}
$$


where

$$
S_{3}:=(-1)^{r} \sum_{p \in \mathscr{P}} \frac{\log p}{p} \widehat{\Phi}\left(\frac{\log \left(p^{2}\right)}{\log \left(q^{r}\right)}\right) .
$$

By partial summation,

$$
S_{3}=(-1)^{r} \int_{1}^{+\infty} \frac{\theta(t)}{t^{2}}\left(\widehat{\Phi}\left(\frac{2 \log t}{\log \left(q^{r}\right)}\right)-\frac{2}{\log \left(q^{r}\right)} \widehat{\Phi}^{\prime}\left(\frac{2 \log t}{\log \left(q^{r}\right)}\right)\right) d t .
$$

Then

$$
\begin{aligned}
S_{3}= & (-1)^{r} \int_{1}^{+\infty}\left(\widehat{\Phi}\left(\frac{2 \log t}{\log \left(q^{r}\right)}\right)-\frac{2}{\log \left(q^{r}\right)} \widehat{\Phi}^{\prime}\left(\frac{2 \log t}{\log \left(q^{r}\right)}\right)\right) \frac{d t}{t} \\
& +(-1)^{r} \int_{1}^{+\infty} \frac{\theta(t)-t}{t}\left(\widehat{\Phi}\left(\frac{2 \log t}{\log \left(q^{r}\right)}\right)-\frac{2}{\log \left(q^{r}\right)} \widehat{\Phi}^{\prime}\left(\frac{2 \log t}{\log \left(q^{r}\right)}\right)\right) \frac{d t}{t} .
\end{aligned}
$$

Using the Prime Number Theorem in the totally explicit form

$$
\theta(t)=t+O\left(t e^{-c \sqrt{\log t}}\right)
$$

for some absolute constant $c>0$ and the facts that $\widehat{\Phi}(u)=\widehat{\Phi}(0)+O\left(u^{2}\right)$ and $\widehat{\Phi}^{\prime}(u) \ll|u|$, we get

$$
\begin{aligned}
S_{3}= & (-1)^{r} \frac{\log \left(q^{r}\right)}{2} \int_{0}^{+\infty} \widehat{\Phi}(u) d u-(-1)^{r} \int_{0}^{+\infty} \widehat{\Phi}^{\prime}(u) d u \\
& +(-1)^{r} \widehat{\Phi}(0) \int_{1}^{+\infty} \frac{\theta(t)-t}{t^{2}} d t+O\left(\frac{1}{\log ^{2}\left(q^{r}\right)}\right)
\end{aligned}
$$

and finally

$$
\begin{aligned}
S_{3}= & (-1)^{r} \frac{\log \left(q^{r}\right)}{4} \Phi(0)+(-1)^{r} \widehat{\Phi}(0)\left(1+\int_{1}^{+\infty} \frac{\theta(t)-t}{t^{2}} d t\right) \\
& +O\left(\frac{1}{\log ^{2}\left(q^{r}\right)}\right) .
\end{aligned}
$$

Equations (3.3) and (3.2) entail that

$$
\begin{aligned}
Q= & P_{q}^{1}[\Phi ; r](f)+\sum_{m=0}^{r-1}(-1)^{m} P_{q}^{2}[\Phi ; r, m](f) \\
& -\frac{2}{\log \left(q^{r}\right)} \sum_{\substack{p \in \mathscr{P} \\
p \nmid q}} \sum_{n \geqslant 3} S\left(\alpha_{f}(p) ; n, r\right) \frac{\log p}{p^{n / 2}} \widehat{\Phi}\left(\frac{\log \left(p^{n}\right)}{\log \left(q^{r}\right)}\right) \\
& +\frac{(-1)^{r+1}}{2} \Phi(0)+2(-1)^{r+1} C_{\mathrm{PNT}} \frac{\widehat{\Phi}(0)}{\log \left(q^{r}\right)}+O\left(\frac{1}{\log ^{3}\left(q^{r}\right)}\right) .
\end{aligned}
$$


According to 2.1) and 2.7), we have

$$
S\left(\alpha_{f}(p) ; n, r\right)=\delta_{2 \mid r}+\sum_{\substack{1 \leqslant j \leqslant r \\ j \equiv r(\bmod 2)}}\left[\lambda_{f}\left(p^{j n}\right)-\lambda_{f}\left(p^{j n-2}\right)\right] .
$$

Since $\widehat{\Phi}(u)=\widehat{\Phi}(0)+O\left(u^{2}\right)$, the contribution of $\delta_{2 \mid r}$ leads to

$$
\begin{aligned}
\sum_{\substack{p \in \mathscr{P} \\
p \nmid q}} \sum_{n \geqslant 3} \frac{\log p}{p^{n / 2}} \widehat{\Phi}\left(\frac{n \log p}{\log \left(q^{r}\right)}\right) & =\sum_{p \in \mathscr{P}} \sum_{n \geqslant 3} \frac{\log p}{p^{n / 2}} \widehat{\Phi}(0)+O\left(\frac{1}{\log ^{2}\left(q^{r}\right)}\right) \\
& =\sum_{p \in \mathscr{P}} \frac{\log p}{p^{3 / 2}-p} \widehat{\Phi}(0)+O\left(\frac{1}{\log ^{2}\left(q^{r}\right)}\right) .
\end{aligned}
$$

According to the previous estimate and equation (3.4), we get

$$
\begin{aligned}
& Q=P_{q}^{1}[\Phi ; r](f)+\sum_{m=0}^{r-1}(-1)^{m} P_{q}^{2}[\Phi ; r, m](f)+P_{q}^{3}[\Phi ; r](f) \\
& +\frac{(-1)^{r+1}}{2} \Phi(0)+2(-1)^{r+1} C_{\mathrm{PNT}} \frac{\widehat{\Phi}(0)}{\log \left(q^{r}\right)}-2 \delta_{2 \mid r} C \frac{\widehat{\Phi}(0)}{\log \left(q^{r}\right)}+O\left(\frac{1}{\log ^{3}\left(q^{r}\right)}\right) .
\end{aligned}
$$

Finally, the result is deduced from this equation for $Q$ and (3.1).

4. Proof of Theorem A. The aim of this part is to determine an asymptotic expansion of

$$
\sum_{f \in H_{\kappa}^{*}(q)} \omega_{q}(f) D_{1, q}[\Phi ; r](f)=: \mathbb{E}_{q}^{\mathrm{h}}\left(D_{1, q}[\Phi ; r]\right) .
$$

According to Proposition 3.1 and the proof of [22, (4.6) and (4.7)], if

$$
\nu<\left(1-\frac{1}{2(\kappa-2 \theta)}\right) \frac{2}{r^{2}}
$$

then

$$
\begin{aligned}
\mathbb{E}_{q}^{\mathrm{h}}\left(D_{1, q}[\Phi ; r]\right)= & {\left[\widehat{\Phi}(0)+\frac{(-1)^{r+1}}{2} \Phi(0)\right] } \\
& +\frac{\widehat{\Phi}(0)}{\log \left(q^{r}\right)}\left[C_{\infty}+2(-1)^{r+1} C_{\mathrm{PNT}}-2 \delta_{2 \mid r} C\right] \\
& +\mathbb{E}_{q}^{\mathrm{h}}\left(P_{q}^{3}[\Phi ; r](f)\right)+O\left(\frac{1}{\log ^{3}\left(q^{r}\right)}\right) .
\end{aligned}
$$

The first term in 4.2 is the main term given in Theorem A. We now estimate the penultimate term of 4.2 via the Petersson trace formula given in [22, Proposition 2.2]. For completeness, we recall that this formula uses 
the $\Delta$ symbol defined for any integers $l, m$ and $n$ by

$$
\Delta_{l}(m, n):=\delta(m, n)+2 \pi i^{\kappa} \sum_{\substack{c \geqslant 1 \\ l \mid c}} \frac{S(m, n ; c)}{c} J_{\kappa-1}\left(\frac{4 \pi \sqrt{m n}}{c}\right)
$$

where $\delta(m, n)$ is 1 if $m=n$ and 0 otherwise, $S(m, n ; c)$ is a Kloosterman sum and $J_{\kappa-1}$ is the Bessel $J$ function. The Petersson trace formula leads to

$$
\mathbb{E}_{q}^{\mathrm{h}}\left(P_{q}^{3}[\Phi ; r]\right)=\mathbb{P}_{q, \text { new }}^{3}[\Phi ; r]+\mathbb{P}_{q, \text { old }}^{3}[\Phi ; r]
$$

where

and

$$
\begin{array}{r}
\mathbb{P}_{q, \text { new }}^{3}[\Phi ; r]=-\frac{2}{\log \left(q^{r}\right)} \sum_{\substack{p \in \mathscr{P} \\
p \nmid q}} \sum_{n \geqslant 3}\left[\sum_{\substack{1 \leqslant j \leqslant r \\
j \equiv r(\bmod 2)}}\left(\Delta_{q}\left(p^{j n}, 1\right)-\Delta_{q}\left(p^{j n-2}, 1\right)\right)\right] \\
\times \frac{\log p}{p^{n / 2}} \widehat{\Phi}\left(\frac{n \log p}{\log \left(q^{r}\right)}\right)
\end{array}
$$

$$
\begin{aligned}
& \mathbb{P}_{q, \text { old }}^{3}[\Phi ; r]=\frac{2}{q \log \left(q^{r}\right)} \sum_{l \mid q^{\infty}} \frac{1}{l} \\
& \quad \times \sum_{\substack{p \in \mathscr{P} \\
p \nmid q}} \sum_{n \geqslant 3}\left[\sum_{\substack{1 \leqslant j \leqslant r \\
j \equiv r(\bmod 2)}}\left(\Delta_{1}\left(p^{j n} l^{2}, 1\right)-\Delta_{1}\left(p^{j n-2} l^{2}, 1\right)\right)\right] \frac{\log p}{p^{n / 2}} \widehat{\Phi}\left(\frac{n \log p}{\log \left(q^{r}\right)}\right) .
\end{aligned}
$$

Let us estimate the new part which can be written as

$$
\begin{aligned}
& \mathbb{P}_{q, \text { new }}^{3}[\Phi ; r] \\
& \quad=-\frac{2\left(2 \pi i^{\kappa}\right)}{\log \left(q^{r}\right)} \sum_{\substack{1 \leqslant j \leqslant r \\
j \equiv r(\bmod 2)}} \sum_{n \geqslant 3}\left(\mathbb{P}_{q, \text { new }}^{3}[\Phi ; r, j n]-\mathbb{P}_{q, \text { new }}^{3}[\Phi ; r, j n-2]\right)
\end{aligned}
$$

where

$$
\begin{aligned}
\mathbb{P}_{q, \text { new }}^{3}[\Phi ; r, k]:= & \sum_{\substack{p \in \mathscr{P} \\
p \neq q}} \frac{\log p}{p^{n / 2}} \widehat{\Phi}\left(\frac{\log p}{\log \left(q^{r / n}\right)}\right) \\
& \times \sum_{\substack{c \geqslant 1 \\
q \mid c}} \frac{S\left(p^{k}, 1 ; c\right)}{c} J_{\kappa-1}\left(\frac{4 \pi \sqrt{p^{k}}}{c}\right) .
\end{aligned}
$$

By [22, Lemma 3.10], the $c$-sum in 4.3 is bounded by

$$
\frac{\tau(q)}{\sqrt{q}} \begin{cases}\left(\frac{\sqrt{p^{k}}}{q}\right)^{1 / 2} & \text { if } p>q^{2 / k} \\ \left(\frac{\sqrt{p^{k}}}{q}\right)^{\kappa-1} & \text { otherwise. }\end{cases}
$$


We deduce

$$
\begin{aligned}
\sum_{n \geqslant 3} \mathbb{P}_{q, \text { new }}^{3}[\Phi ; r, j n] & \ll \frac{\tau(q)}{q^{\kappa-1 / 2}} \sum_{n \geqslant 3} \sum_{p \leqslant q^{r \nu / n}} \frac{1}{p^{n / 2}} p^{r n(\kappa-1) / 2} \log p \\
& \ll \frac{\tau(q)}{q^{\kappa-1 / 2}} \sum_{3 \leqslant n \leqslant \nu r \log q / \log 2} \frac{1}{n} q^{\nu r[((\kappa-1) r-1) n / 2+1] / n} \\
& \ll \frac{\tau(q)}{q^{\kappa-1 / 2}} q^{\nu r[(\kappa-1) r-1] / 2} q^{\nu r / 3} \log \log (3 q) \ll \frac{1}{q^{1 / 2}}
\end{aligned}
$$

as soon as $\nu<2 / r^{2}$ (and in particular if (4.1) is satisfied). We make the same computations for $j n-2$ and find then that $\mathbb{P}_{q, \text { new }}^{3}[\Phi ; r, k]$ is an admissible error term. The old part is

$$
\mathbb{P}_{q, \text { old }}^{3}[\Phi ; r]=\frac{2\left(2 \pi i^{\kappa}\right)}{q \log \left(q^{r}\right)} \sum_{\substack{1 \leqslant j \leqslant r \\ j \equiv r(\bmod 2)}} \sum_{n \geqslant 3}\left(\mathbb{P}_{q, \text { old }}^{3}[\Phi ; r, j n]-\mathbb{P}_{q, \text { old }}^{3}[\Phi ; r, j n-2]\right)
$$

where

$$
\mathbb{P}_{q, \text { old }}^{3}[\Phi ; r, k]:=\sum_{\substack{p \in \mathscr{P} \\ p \neq q}} \frac{\log p}{p^{n / 2}} \widehat{\Phi}\left(\frac{\log p}{\log \left(q^{r / n}\right)}\right) \sum_{l \mid q^{\infty}} \frac{1}{l} \Delta_{1}\left(p^{k} l^{2}, 1\right) .
$$

From [22, (3.2) and (3.3)] we have

$$
\sum_{l \mid q^{\infty}} \frac{1}{l} \Delta_{1}\left(p^{k} l^{2}, 1\right) \leqslant 2(k+1)
$$

so that

$$
\sum_{n \geqslant 3} \mathbb{P}_{q, \text { old }}^{3}[\Phi ; r, j n] \ll 1
$$

and similarly for $\mathbb{P}_{q, \text { old }}^{3}[\Phi ; r, j n-2]$. Thus, $\mathbb{E}_{q}^{\mathrm{h}}\left(P_{q}^{3}[\Phi ; r]\right)$ enters the error term of size $O\left(1 / \log ^{3}\left(q^{r}\right)\right)$.

Appendix A. Some comments on an aesthetic identity. It is possible to prove by induction on $k_{0} \geqslant 1$ the following equality in $\mathbb{Q}[T]$ :

$$
\begin{aligned}
& X_{2 k_{0}}-X_{2 k_{0}-2} \\
& =\sum_{j=0}^{k_{0}-1} \sum_{1 \leqslant k_{j}<k_{j-1}<\cdots<k_{1}<k_{0}}(-1)^{j}\left[\prod_{i=0}^{j-1}\left(\begin{array}{c}
2 k_{i} \\
k_{i}-k_{i+1}
\end{array}\right)\right]\left\{T^{2 k_{j}}-\left(\begin{array}{c}
2 k_{j} \\
k_{j}
\end{array}\right)\right\} .
\end{aligned}
$$

As a consequence, if $K \geqslant 1$ then

$$
X_{2 K+1}-X_{2 K-1}=(-1)^{K} T\left(1+\sum_{1 \leqslant k_{0} \leqslant K}(-1)^{k_{0}} X_{2 k_{0}}-X_{2 k_{0}-2}\right) .
$$


Now, use 2.4 with $r=1$ (so that $X_{1}=T$ ) to get from A.1) the equality $X_{2 k_{0}}-X_{2 k_{0}-2}$

$=\sum_{j=0}^{k_{0}-1} \sum_{1 \leqslant k_{j}<k_{j-1}<\cdots<k_{1}<k_{0}}(-1)^{j}\left[\prod_{i=0}^{j-1}\left(\begin{array}{c}2 k_{i} \\ k_{i}-k_{i+1}\end{array}\right)\right]\left[\sum_{l=0}^{2 k_{j}} x\left(2 k_{j}, 1, l\right) X_{l}-\left(\begin{array}{c}2 k_{j} \\ k_{j}\end{array}\right) X_{0}\right]$ and compare the coefficients of $X_{0}$ to obtain, thanks to (2.5), the equality

$$
\sum_{j=0}^{k_{0}-1} \sum_{1 \leqslant k_{j}<k_{j-1}<\cdots<k_{1}<k_{0}}(-1)^{j}\left[\prod_{i=0}^{j-1}\left(\begin{array}{c}
2 k_{i} \\
k_{i}-k_{i+1}
\end{array}\right)\right]\left(\begin{array}{c}
2 k_{j} \\
k_{j}
\end{array}\right) \frac{k_{j}}{1+k_{j}}=0 .
$$

We could have expressed formulas $(A .1)$ and $(A .2)$ in terms of Fourier coefficients of primitive forms to determine the lower order terms. However, this is definitely not the best way to proceed since it consists in decomposing the polynomial $X_{K}-X_{K-2}$ in the canonical basis of $\mathbb{Q}[T]$ and decomposing again each element of this canonical basis in the Chebyshev basis $\left(X_{l}\right)_{l \in \mathbb{N}}$.

\section{Appendix B. S. J. Miller's identity and Chebyshev polynomi-} als. S. J. Miller [18, (3.12), p. 6] recently proved that

$$
\alpha_{f}(p)^{K}+\beta_{f}(p)^{K}=\sum_{\substack{0 \leqslant k \leqslant K \\ k \equiv K(\bmod 2)}} c_{K, k} \lambda_{f}(p)^{k}
$$

where $c_{K, k}=0$ if $k \equiv K+1(\bmod 2)$ and

$$
\begin{aligned}
c_{0,0} & =0, \\
c_{2 K, 0} & =2(-1)^{K} \quad(K \geqslant 1), \\
c_{2 K, 2 L} & =\frac{2(-1)^{K+L} K(K+L-1) !}{(2 L) !(K-L) !} \quad(1 \leqslant L \leqslant K), \\
c_{2 K+1,2 L+1} & =\frac{(-1)^{K+L}(2 K+1)(K+L) !}{(2 L+1) !(K-L) !} \quad(0 \leqslant L \leqslant K) .
\end{aligned}
$$

We would like to give a quick proof of this identity, the crucial tool being Chebyshev polynomials.

Proof of equation (B.1). We know that

$$
\alpha_{f}(p)^{K}+\beta_{f}(p)^{K}=X_{K}\left(\lambda_{f}(p)\right)-X_{K-2}\left(\lambda_{f}(p)\right)
$$

for $K \geqslant 2$ according to 2.6). Thus, the proof consists in decomposing the polynomial $X_{K}-X_{K-2}$ in the canonical basis of $\mathbb{Q}[T]$. This can be done via 2.9. It entails that 


$$
\begin{gathered}
\alpha_{f}(p)^{K}+\beta_{f}(p)^{K}=\sum_{\substack{K-1 \leqslant u \leqslant K \\
u \equiv K(\bmod 2)}}(-1)^{(K-u) / 2}\left(\begin{array}{c}
(K+u) / 2 \\
u
\end{array}\right) \lambda_{f}(p)^{u} \\
+\sum_{\substack{0 \leqslant u \leqslant K-2 \\
u \equiv K(\bmod 2)}}(-1)^{(K-u) / 2}\left[\left(\begin{array}{c}
(K+u) / 2 \\
u
\end{array}\right)+\left(\begin{array}{c}
(K+u) / 2-1 \\
u
\end{array}\right)\right] \lambda_{f}(p)^{u},
\end{gathered}
$$

which is an equivalent formulation of (B.1).

REMARK B.1. Equation B.1 could be used to recover the lower order terms coming from $P_{q}^{3}[\Phi ; r]$ but, once again, it is not the most clever way to proceed since it would imply decomposing the polynomials $X_{K}-X_{K-2}$ in the canonical basis of $\mathbb{Q}[T]$ at the beginning of the process and decomposing the polynomials $T^{j}$ in the basis $\left(X_{r}\right)_{r \geqslant 0}$ just before the end of the proof in order to be able to apply some trace formula for the Fourier coefficients of cusp forms.

Acknowledgements. The first author is financed by the ANR project "Aspects Arithmétiques des Matrices Aléatoires et du Chaos Quantique". He would like to thank the University of Nottingham, where this work was finished, for its hospitality. The second one is supported by the ANR project "Modunombres" and the Blaise Pascal University project "Nomex". Both authors would like to thank Karim Belabas for a fruitful discussion on numerical evaluations of constants and the anonymous referee for his comments.

\section{References}

[1] T. Barnet-Lamb, D. Geraghty, M. Harris, and R. Taylor, A family of Calabi-Yau varieties and potential automorphy II, preprint, 2009: http://www.math.harvard. edu/ $\sim$ rtaylor $/$.

[2] C. Batut, K. Belabas, D. Benardi, H. Cohen and M. Olivier, User's Guide to PARI$G P$, http://pari.math.u-bordeaux1.fr/.

[3] D. Broadhurst, unpublished, http://physics.open.ac.uk/ dbroadhu/cert/cohenb3.ps (2005).

[4] J. Cogdell and P. Michel, On the complex moments of symmetric power L-functions at $s=1$, Int. Math. Res. Notices 2004, no. 31, 1561-1617.

[5] H. Cohen, High precision computation of Hardy-Littlewood constants, unpublished, http://www.math.u-bordeaux.fr/ ${ }^{\sim}$ cohen/.

[6] E. Fouvry and H. Iwaniec, Low-lying zeros of dihedral L-functions, Duke Math. J. 116 (2003), 189-217.

[7] S. Gelbart and H. Jacquet, A relation between automorphic representations of GL(2) and GL(3), Ann. Sci. École Norm. Sup. (4) 11 (1978), 471-542.

[8] I. S. Gradshteyn and I. M. Ryzhik, Tables of Integrals, Series, and Products, 7th ed., Elsevier/Academic Press, Amsterdam, 2007. 
[9] A. M. Güloğlu, Low-lying zeroes of symmetric power L-functions, Int. Math. Res. Notices 2005, no. 9, 517-550.

[10] E. Hecke, Über die Bestimmung Dirichletscher Reihen durch ihre Funktionalgleichung, Math. Ann. 112 (1936), 664-699.

[11] —, Über Modulfunktionen und die Dirichletschen Reihen mit Eulerscher Produktentwicklung. I, ibid. 114 (1937), 1-28.

[12] —, Über Modulfunktionen und die Dirichletschen Reihen mit Eulerscher Produktentwicklung. II, ibid. 114 (1937), 316-351.

[13] H. Iwaniec, W. Z. Luo and P. Sarnak, Low lying zeros of families of L-functions, Inst. Hautes Études Sci. Publ. Math. 91 (2000), 55-131.

[14] H. H. Kim, Functoriality for the exterior square of $\mathrm{GL}_{4}$ and the symmetric fourth of $\mathrm{GL}_{2}$, J. Amer. Math. Soc. 16 (2003), 139-183, with appendix 1 by D. Ramakrishnan and appendix 2 by H. H. Kim and P. Sarnak.

[15] H. H. Kim and F. Shahidi, Cuspidality of symmetric powers with applications, Duke Math. J. 112 (2002), 177-197.

[16] -, - Functorial products for $\mathrm{GL}_{2} \times \mathrm{GL}_{3}$ and the symmetric cube for $\mathrm{GL}_{2}$, Ann. of Math. (2) 155 (2002), 837-893, with an appendix by C. J. Bushnell and G. Henniart.

[17] S. J. Miller, An orthogonal test of the L-functions ratio conjecture, Proc. London Math. Soc. (3) 99 (2009), 484-520.

[18] —, An identity for sums of polylogarithm functions, Integers 8 (2008), A15, 10 pp.

[19] - A symplectic test of the L-functions ratios conjecture, Int. Math. Res. Notices 2008, no. 3, art. ID rnm146, 36 pp.

[20] - , Lower order terms in the 1-level density for families of holomorphic cuspidal newforms, Acta Arith. 137 (2009), 51-98.

[21] G. Ricotta, Real zeros and size of Rankin-Selberg L-functions in the level aspect, Duke Math. J. 131 (2006), 291-350.

[22] G. Ricotta and E. Royer, Statistics for low-lying zeros of symmetric power Lfunctions in the level aspect, submitted, 2007, http://arxiv.org/abs/math/0703760.

[23] J. B. Rosser and L. Schoenfeld, Approximate formulas for some functions of prime numbers, Illinois J. Math. 6 (1962), 64-94.

[24] E. Royer and J. Wu, Special values of symmetric power L-functions and Hecke eigenvalues, J. Théor. Nombres Bordeaux 19 (2007), 703-753.

[25] W. Rudin, Analyse fonctionnelle, Ediscience international, Paris, 1995.

[26] M. P. Young, Lower-order terms of the 1-level density of families of elliptic curves, Int. Math. Res. Notices 2005, no. 10, 587-633.

Guillaume Ricotta

Laboratoire A2X

Université de Bordeaux 1

351, cours de la Libération

F-33405 Talence Cedex, France

E-mail: Guillaume.Ricotta@math.u-bordeaux1.fr
Emmanuel Royer

Laboratoire de mathématiques

Clermont Université

Université Blaise Pascal

BP 10448

F-63000 Clermont-Ferrand, France

Current address:

CNRS, UMR 6620

Laboratoire de mathématiques

F-63177 Aubière, France

E-mail: emmanuel.royer@m4x.org 